\title{
ON EMBEDDING MANIFOLDS WHICH ARE BUNDLES OVER SPHERES ${ }^{1}$
}

\author{
MARK MAHOWALD
}

1. Statement of results. In this note we will prove the following theorem.

THEOREM 1.1. Suppose $M$ is the total space of a fiber bundle over $S^{n}$ with fiber $F, a$ compact, $k$-dimensional $\pi$-manifold. If $F$ embeds in $R^{k+m}$ with a normal field of $n$ frames, then $M$ topologically embeds in $R^{2 k+m+2}$ and differentiably embeds in $R^{2 k+m+j}$, where $j \geqq 2$ and $j \geqq(m-k+3) / 2$.

The proof is given in $\$ 4$. By a simplified version of the argument we also can prove

Theorem 1.2. Suppose $M$ and $F$ are as in Theorem 1.1. If $F$ embeds in $R^{k+q}$ with a trivial normal bundle for some $q \leqq n / 2$, then $M$ is topologically embeddable in $R^{2 k+n+1}$ and differentiably embeddable in $R^{2 k+n+j}$, where $j \geqq 1$ and $j \geqq(n-k+3) / 2$.

The proof is given in $\S 3$. We have immediately this

CoRollary 1.3. If $M$ is the total space of an $S^{k}$ bundle over $S^{n}$, then $M$ topologically embeds in $R^{2 k+n+1}$.

These results were discovered in an attempt to embed real projective spaces. The well-known Hopf fiberings of $P_{15}$ and $P_{7}$ give the following representations of these spaces: $P_{7}$ is a fiber space over $S^{4}$ with $P_{3}$ as fiber, and $P_{15}$ is a fiber space over $S^{8}$ with $P_{7}$ as fiber. Since any embedding of $P_{3}$ in $R^{5}$ has a trivial normal bundle [6], Theorem 1.2 applies for $P_{7}$. Theorem 1.1 applies for $P_{15}$ and, hence, we have

Theorem 1.4. There exists a topological embedding of $P_{7}$ into $R^{11}$. There exists a differentiable embedding of $P_{7}$ into $R^{12}$ and of $P_{15}$ into $R^{24}$.

Corollary 1.5. There exists a differentiable embedding of $P_{14}$ into $R^{24}$.

In [5] it is proved that $P_{14}$ embeds in $R_{25}$ and $P_{15}$ embeds in $R^{28}$. These results followed from rather extensive computations of secondary characteristic classes. The proof given here is geometric. James

Received by the editors December 12, 1962 and, in revised form, March 13, 1963.

1 This work was supported by the U.S. Army Research Office (Durham). 
[3] has shown that $P_{15}$ does not immerse in $R_{21}$ and indicates that Sanderson has an immersion of $P_{15}$ in $R_{22}$. It therefore seems likely that this embedding of $P_{15}$ is the best possible. For other recent work on embedding projective spaces, see [1] and [2].

Another application is

TheOREM 1.6. $\mathrm{SO}(5)$ embeds in $R^{17}$.

Proof. Since $\mathrm{SO}(4) \cong \mathrm{SO}(3) \times S^{3}[7,8.6]$ and $\mathrm{SO}(5)$ is an $\mathrm{SO}(4)$ bundle over $S^{4}$, Theorem 1.2 applies.

We wish to thank W. S. Massey for his encouragement and help during the preparation of this paper.

\section{A lemma.}

2.1. Let $g: F \times S^{n-1} \rightarrow F$ and let $Y=\left(F \times D^{n}\right) \cup_{g} F$. We can represent points in $Y$ by triplets $(f, s, r)$, where $f \in F, s \in S^{n-1}$ and $0 \leqq r \leqq 1$, under the equivalence relation $(f, s, r) \sim\left(f^{\prime}, s^{\prime}, r^{\prime}\right)$ iff

$$
\begin{gathered}
r=r^{\prime}=0 \text { and } \quad f=f^{\prime} ; \text { or } \\
r=r^{\prime}=1 \text { and } g(f, s)=g\left(f^{\prime}, s^{\prime}\right) ; \text { or } \\
(f, s, r)=\left(f^{\prime}, s^{\prime}, r^{\prime}\right) .
\end{gathered}
$$

Let $h_{1}: F \times D^{n} \rightarrow R^{q}$ and $h_{2}: F \rightarrow S^{p} \subset R^{p+1}$ be embeddings. An embedding of $Y$ in $R^{q+p+1}$ can be realized by joining $h_{1}(f, s, 1)$ and $h_{2}(g(f, s))$ with a line segment. This topological embedding is essentially given by

$$
(f, s, r) \rightarrow\left((1-r) h_{1}(f, s, r), r h_{2}(g(f, s))\right) .
$$

This embedding is, in general, not very good since the full strength of $h_{2}$ is needed only when $r=1$ and for this value of $r, h_{1}$ is not needed. Lemma 2.2 states a weaker condition under which we still get an embedding.

Lemma 2.2. Let $F$ be a compact space and let $D^{n}$ be the unit ball in $R^{n}$. Suppose there exists a map I: $F \times F \times D^{n} \rightarrow R^{i}$ such that:

(i) If $|x|=0$ or 1 , then $I\left(f_{1}, f_{2}, x\right)$ is independent of $f_{i}$, where $i=1+|x|$.

(ii) If $|x|=1$, then $I$ does not depend on $x$.

(iii) If $|x|<1,|x|=\left|x^{\prime}\right|$ and $I\left(f_{1}, f_{2}, x\right)=I\left(f_{1}^{\prime}, f_{2}^{\prime}, x^{\prime}\right)$, then $f_{2}=f_{2}^{\prime}$ and $x=x^{\prime}$

(iv) If $|x|=1=\left|x^{\prime}\right|$ and $I\left(f_{1}, f_{2}, x\right)=I\left(f_{1}^{\prime}, f_{2}^{\prime}, x^{\prime}\right)$, then $f_{1}=f_{1}^{\prime}$. Then the total space $M$ of any bundle over $S^{n}$ with fiber $F$ is topologically embeddable in $R^{j+1}$. 
Proof. Let $s$ be any point in $S^{n}$ and let $p: M \rightarrow S^{n}$ be the fiber map. Then $M-p^{-1}(s)$ is homeomorphic to $F \times R^{n}$. There exists a map $g: F \times S^{n-1} \rightarrow F$ such that $\left(F \times D^{n}\right) \cup{ }_{0} F$ is homeomorphic to $M$, hence, we can represent $M$ by ordered pairs $(f, x)$ where $f \in F$ and $|x|<1$ plus $(f, x)$ where $|x|=1$ modulo the equivalence relation $(f, x)$ $\sim\left(f^{\prime}, x^{\prime}\right)$ iff $g(f, x)=g\left(f^{\prime}, x^{\prime}\right)$. Then the map $J: M \rightarrow R^{j} \times R^{1}$ defined by

$$
J(f, x)=\left(I\left(g\left(f, \frac{x}{|x|}\right), f, x\right),|x|\right)
$$

is the desired embedding. First observe that when $x=0, I$ is independent of $f_{1}$ by (i) and, hence, the apparent singularity in the first coordinate of the argument of $I$ does not occur. Now suppose $J(f, x)$ $=J\left(f^{\prime}, x^{\prime}\right)$. Then clearly $|x|=\left|x^{\prime}\right|$. Suppose $|x|<1$. By (iii) $f=f^{\prime}$ and $x=x^{\prime}$. If $|x|=1$ then, by (iv), $g(f, x)=g\left(f^{\prime}, x^{\prime}\right)$ and this is just what is required for $M$.

3. Proof of Theorem 1.2. The hypotheses of Theorem $1.2 \mathrm{imply}$ that there exist integers $n_{1}$ and $n_{2}$ and maps $\alpha_{1}$ and $\alpha_{2}$ such that $n_{1}+n_{2}=n$ and $\alpha_{i}: F \times R^{n_{i}} \rightarrow R^{k+n_{i}}$. Let points $x \in R^{n}$ be written as pairs $(y, z)$ with $y \in R^{n_{1}}$ and $z \in R^{n_{2}}$. Let

$$
I\left(f_{1}, f_{2},(y, z)\right)=\left(r \alpha_{1}\left(f_{1},(1-r) y\right),(1-r) \alpha_{2}\left(f_{2}, z\right)\right),
$$

where $r=|(y, z)|$. Clearly $I$ satisfies Lemma 2.1 and the topological portion of the theorem is proved.

The differentiable part follows from the topological part and this theorem of Haefliger [4]:

THEOREM (HAEFLIGER). If there exists a topological embedding of an $n$-dimensional manifold $M$ into $R^{k}$ and if $2 k \geqq 3(n+1)$, then there exists a differentiable embedding of $M$ into $R^{k}$.

\section{Proof of Theorem 1.1.}

4.1. We will first give a heuristic description of the proof. The details are given in 4.2 . Since $F$ is a $\pi$-manifold the normal bundle to any embedding of $F$ in $R^{p}, p \geqq 2 k+1$, will be trivial. In particular $F \times R^{k+m}$ embeds in $R^{2 k+m}$. Using this we can get an embedding of $F \times F \times D^{n}$ into $R^{2 k+m}$. Because the factor $F \times D^{n}$ is embedded in the normal bundle to the embedding of $F$ we can not use a simple formula like 2.1.2 or even the idea of 3.1 to get an embedding of $M$. What we do is to construct a homotopy $h_{t}^{\prime}: F \times R^{k+m} \rightarrow R^{k+1} \times R^{k+m}$ based on a regular homotopy of an immersion of $F$ in $R^{k+1}$ into an embedding of $F$ during which a normal field of $k+m$ frames is dragged along. This regular homotopy is then modified so that: (1) $h_{0}^{\prime}$ maps the 
second factor in a 1-1 fashion and maps the first factor trivially; (2) $h_{1}^{\prime}$ is an embedding of $F \times R^{k+m}$ into $R^{2 k+m+1}$; (3) if $i_{f}: R^{k+m}$ $\rightarrow F \times R^{k+m}$ is defined by $i_{f}(x) \rightarrow(f, x)$, then $h_{t}^{\prime} i_{f}$ is an embedding for each $f$ and each $t$. Let $\gamma: F \times D^{n} \rightarrow R^{k+m}$ be the embedding given by the hypothesis of the theorem. The map'

$$
I\left(f_{1}, f_{2}, x\right)=h_{|x|}^{\prime}\left(f_{1},(1-|x|) \gamma\left(f_{2}, x\right)\right)
$$

will satisfy Lemma 2.2 .

4.2. Since $F$ is a $\pi$-manifold, $F$ can be immersed in $R^{k+1}$. Let $\alpha$ be such an immersion and let $\beta$ be an embedding of $F$ into $R^{k+m}$. Then $h_{1}: F \rightarrow R^{2 k+m+1}$, defined by $h_{1}(f)=(\alpha(f), \beta(f)) \in R^{k+1} \times R^{k+m}$, is an embedding of $F$ into $R^{2 k+m+1}$. Let $h_{t}: F \rightarrow R^{2 k+m+1}$ be defined by $h_{t}(f)$ $=(\alpha(f),(2 t-1) \beta(f))$ for $\frac{1}{2} \leqq t \leqq 1$. This is a regular homotopy of the embedding $h_{1}$. Let $\hat{h}_{t}: F \rightarrow G_{k, k+m+1}$ be the map which assigns to each point in $F$ the $k+m+1$ plane in $R^{2 k+m+1}$ which is normal to $F$ at the point. Since $h_{t}$ is a regular homotopy it is clear that $\hat{h}_{t}$ is a homotopy. Let $p: E \rightarrow G_{k, k+m+1}$ be the universal $V_{1, k+m}$ bundle. ${ }^{2}$ (The space $E$ can be thought of as the collection of pairs $\left\{\left(\right.\right.$ a $k+m+1$ plane in $R^{2 k+m+1}$, a $k+m$ frame in that $k+m+1$ plane) $\}$.) Let $\hat{H}_{1 / 2}: F \rightarrow E$ be the map which assigns to each point of $F$ the $k+m$ frame in the normal $k+m+1$ plane consisting of the unit vectors formed by the last $k+m$ coordinates of $R^{2 k+m+1}$. Clearly $p \hat{H}_{1 / 2}=\hat{h}_{1 / 2}$. By the $H C P$ we can extend $\hat{H}_{1 / 2}$ to a homotopy $\hat{H}_{t}: F \rightarrow E$ which covers $\hat{h}_{t}$. Let $i: E \rightarrow V_{k+1, k+m}$ be the map which assigns to each $k+m$ frame in a $k+m+1$ subplane of $R^{2 k+m+1}$ the same frame considered as a $k+m$ frame in $R^{2 k+m+1}$. Let $H_{t}=i \hat{H}_{t}$.

We can think of each point in $V_{k+1, k+m}$ as an ordered set of $k+m$ linearly independent vectors in $R^{2 k+m+1}$. Let $H_{t}^{i}(f)$ be the $i$ th vector of $H_{t}(f)$. Since $F$ is compact we can choose $H_{t}$ so that if $x \in D^{k+m}$, the unit disk in $R^{k+m}$, then $(f, x) \rightarrow \sum_{i=1}^{k+m} H_{t}^{i}(f) x_{i}+h_{t}(f)$ is an embedding of $F \times D^{k+m}$ for each $t>\frac{1}{2}$.

Clearly $H_{1 / 2}$ is a constant map and we define $H_{t}=H_{1 / 2}$ for $t \leqq \frac{1}{2}$. Let $h_{t}(f)=(2 t \alpha(f), 0)$ for $t \leqq \frac{1}{2}$. Then

$$
\sum_{i=1}^{k+m} H_{t}^{i}(f) x_{i}+h_{t}(f)=(2 t \alpha(f), x) \quad \text { if } t \leqq \frac{1}{2} .
$$

The homotopy $h_{t}^{\prime}: F \times D^{k+m} \rightarrow R^{2 k+m+1}$ is defined by

$$
h_{t}^{\prime}(f, x)=\sum_{i=1}^{k+m} H_{t}^{i}(f) x_{i}+h_{t}(f) .
$$

\footnotetext{
2 In this section we wish to consider $V_{k, n}$ as the collection of $n$ frames in $R^{k+n}$ which are not necessarily orthonormal.
} 
Let $\gamma: F \times R^{n} \rightarrow R^{k+m}$ be the embedding whose existence follows from the embedding of $F$ into $R^{k+m}$ with a normal field of $n$-frames. Choose $\gamma$ so that $|\gamma| \leqq 1$. Let $\gamma_{i}(f, x)$ be the $i$ th coordinate of $\gamma(f, x)$. Let $I: F \times F \times D^{n} \rightarrow R^{2 k+m+1}$ be defined by

(4.2.3) $I\left(f_{1}, f_{2}, x\right)=\sum_{i=1}^{m+k}(1-|x|) H_{|x|}^{i}\left(f_{1}\right) \gamma_{i}\left(f_{2}, x\right)+h_{|x|}\left(f_{1}\right)$.

It is easily verified that $I$ satisfies the conditions of Lemma 2.2. This proves the topological part. The differentiable part follows as in $\S 3$.

\section{REFERENCES}

1. D. B. Epstein and R. L. Schwarzenberger, Embeddings of real projective spaces, Ann. of Math. (2) 76 (1962), 180-184.

2. I. M. James, Some embeddings of projective spaces, Proc. Cambridge Philos. Soc. 55 (1959), 294-298.

3. - On the immersion problem for real projective spaces, Bull. Amer. Math. Soc. 69 (1963), 231-238.

4. A. Haefliger, Plongements différentiables de variêtês dans variêtés, Comment. Math. Helv. 36 (1961), 47-82.

5. M. E. Mahowald, On extending cross sections in orientable $V_{k+m, m}$ bundles, Bull. Amer. Math. Soc. 68 (1962), 596-602.

6. W. S. Massey, On the normal bundle of a sphere imbedded in Euclidean space, Proc. Amer. Math. Soc. 10 (1959), 959-964.

7. N. Steenrod, Topology of fibre bundles, Princeton Mathematical Series, Vol. 14, Princeton Univ. Press, Princeton, N. J., 1951.

Syracuse UNIVERSITy 